\title{
Highly focused femtosecond laser directed selective boron doping in single SiC nanowire device for $n-p$ conversion
}

Cite as: Appl. Phys. Lett. 115, 133104 (2019); doi: 10.1063/1.5115335

Submitted: 19 June 2019 - Accepted: 6 September 2019 •

Published Online: 23 September 2019
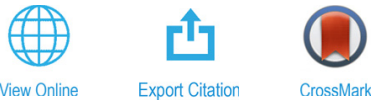

Jinpeng Huo, ${ }^{7}$ (D) Guisheng Zou, ${ }^{7}$ Luchan Lin,, ${ }^{2, a)}$ Kehong Wang, ${ }^{3}$ Songling Xing, ${ }^{1}$ (D) Guanlei Zhao, Lei Liu, ${ }^{1, a)}$ (iD and Y. Norman Zhou ${ }^{1,4}$

\begin{abstract}
AFFILIATIONS
'Department of Mechanical Engineering, State Key Laboratory of Tribology, Key Laboratory for Advanced Manufacturing by Materials Processing Technology, Ministry of Education of PR China, Tsinghua University, Beijing 100084, People's Republic of China

${ }^{2}$ Empa, Swiss Federal Laboratories for Materials Science and Technology, Überlandstrasse 129, 8600 Dübendorf, Switzerland ${ }^{3}$ School of Material Science and Engineering, Nanjing University of Science and Technology, Nanjing 210094, China

${ }^{4}$ Centre for Advanced Materials Joining, University of Waterloo, Waterloo, Ontario N2L 3G1, Canada
\end{abstract}

${ }^{a)}$ Authors to whom correspondence should be addressed: Iuchan.lin@empa.ch and liulei@tsinghua.edu.cn

\begin{abstract}
In this work, site-selective Boron (B) doping in $\mathrm{SiC}$ nanowires has been demonstrated by utilizing focused femtosecond (fs) laser irradiation. Raman spectra and electrical performance indicate that the localized element doping in pristine n-type SiC nanowires can convert the segment into p-type. The formation of crystalline defects and vacancies in nanowires under fs laser irradiation, along with the simultaneous dissociation of the dopant molecules, can accelerate the doping process. Single $\mathrm{SiC}$ nanowire p-n junction and field-effect transistors with a p-type segment have been fabricated based on the pristine n-type nanowire, showing a modified electrical response as a logic gate to programmed voltage signals. This laser controlled selective doping may provide an alternative for precise element doping in semiconductors at the nanoscale, which can be promising for nanoelectronic unit fabrication.
\end{abstract}

Published under license by AIP Publishing. https://doi.org/10.1063/1.5115335

Silicon carbide ( $\mathrm{SiC})$, known as a third-generation semiconductor material, has been extensively used in high-power electronics due to its unique properties such as high critical breakdown electric field, high thermal conductivity, high carrier saturation drift velocity, and excellent chemical and physical stability. ${ }^{1}$ By reducing $\mathrm{SiC}$ material to nanoscale, $\mathrm{SiC}$ nanowire (NW) combines the aforementioned properties with a new quantum size effect, which would provide new alternatives to develop nanoscale electronics and optoelectronics. Recently, NWs have been extensively utilized in the design and fabrication of devices such as field emitters, field effect transistors, photocatalysts, nanodevices, and so on. ${ }^{2-6}$ In particular, element doping in semiconductors presents an effective approach to modify the electrical performance of nanodevices by tuning the majority carrier type and resistivity, which could broadly extend their applications in electronic devices. For instance, with Phosphorous (P) and Nitrogen (N) doping, the resulting n-type $\mathrm{SiC}$ NW networks exhibit excellent field emission (FE) properties with ultralow turn-on fields and a strong dependence on the temperatures. ${ }^{7,8}$ While, with Boron (B) doping, the p-type 3C-SiC NWs would display improved FE performance with low turnon fields, a large field enhancement factor, long-term stability in harsh environments, etc. ${ }^{9}$ Meanwhile, $\mathrm{SiC}$ nanowires demonstrate an efficient activity for visible-light-driven photocatalytic hydrogen production by $\mathrm{B}$ or $\mathrm{N}$ doping. ${ }^{10,11}$ In addition, $\mathrm{SiC}$ nanowires with $\mathrm{p}$ - and/or $\mathrm{n}$-type doping have been applied in the fields of energy storage, electrochemistry, biology, etc.

For bulk materials and thin films, element doping can be achieved by ion-implantation, thermal diffusion, or vapor phase codeposition. ${ }^{16-18}$ However, localized doping at the nanoscale still poses a great challenge. Particularly, precise control of input energy during the doping process is the key limitation to achieve selective doping at the nanoscale. Even though approaches for in situ doping and ion implantation can be realized in nanoscale materials, ${ }^{19-21}$ few work has been reported for localized doping, especially to control the accurate location of dopants at the nanoscale without damaging nanomaterials. ${ }^{22}$ 
Due to the highly spatial and temporal controllability, ultrafast laser sources have been widely used in nanoscale device fabrication. During laser irradiation, thermal melting and phase explosion due to nonlinear optical absorption, delayed Auger heating, and highdensity carrier diffusion can occur in semiconductors. ${ }^{23}$ As a result, numerous crystalline defects are generated even when incident energy is below the damage threshold. Simultaneously, dopant activation/dissociation and crystalline defect implementation can also be realized from the same high intensity laser beam. ${ }^{24-27}$ Hence, the dopants may diffuse into the semiconductor via defects generated by laser irradiation, and spatially selective doping at the nanoscale is expected in semiconductors.

In this work, selective boron (B) doping in $\mathrm{SiC}$ NWs by using focused femtosecond (fs) laser irradiation has been demonstrated. Raman scattering and electrical measurements were conducted to characterize the doping states in $\mathrm{SiC}$ NWs after laser irradiation. SiC nanowire $\mathrm{p}-\mathrm{n}$ junction and field-effect transistors (NWFETs) and single-NW $p-n$ junctions were fabricated to elucidate the doping effects in nanodevice modification. This optical doping technique may provide an alternative for precise element doping in semiconductors at the nanoscale, which can be promising for nanoelectronic unit fabrication.

$\mathrm{SiC}$ NWs (XFNANO Materials, China) were dispersed in de-ionized water and then drop-cast on Au interdigital electrodes. Au electrodes with a $4 \mu \mathrm{m}$ finger spacing on the chip were fabricated by optical lithography and lift-off processes on an oxidized Si substrate $\left.(300 \mathrm{~nm} \mathrm{SiO})_{2}\right)$. The electrodes were rinsed with high purity acetone (99.99\%) and de-ionized water before the $\mathrm{Au} / \mathrm{SiC} \mathrm{NW} / \mathrm{Au}$ bridged circuit was assembled. Sodium borohydride $\left(\mathrm{NaBH}_{4}, 96 \%\right)$ was dissolved in ethanol with a solubility of $25 \%$. Then, $10 \mu$ l solution was pointedly dripped at the $\mathrm{NW}$-electrode bridge and dried in the air. Fs laser pulses $(800 \mathrm{~nm}, 1 \mathrm{kHz})$ generated by a Ti:sapphire mode-locked oscillator with a typical pulse duration of $50 \mathrm{fs}$ were then used for localized irradiation. An objective lens $(\mathrm{NA}=0.4)$ was used to focus the laser beam to a circle area with a diameter of $\sim 800 \mathrm{~nm}$ at normal incidence. The laser radiation time is controlled by a mechanical shutter. The schematic of the experimental setup is shown in Fig. 1(a). The morphology and microstructure of SiC NWs were characterized by X-ray diffraction (XRD, Bruker D8), scanning electron microscopy (SEM, JEOL-7001F-77), and transmission electron microscopy (TEM, JEOL-2100F). A commercially available microRaman spectroscopy system (JY HR-800) coupled with a $633 \mathrm{~nm}$ excitation laser and a spectrometer with the range of $600-1100 \mathrm{~cm}^{-1}$ was used for the Raman scattering spectrum measurement. The electrical characterization of the built single NW devices was implemented with a precise source-meter (Agilent B1500A).

Figure 1(d) shows the XRD spectrum and SEM image of the as-prepared $\mathrm{SiC}$ NWs. The diameter of the $\mathrm{SiC}$ NWs ranges from 50 to $200 \mathrm{~nm}$. The XRD pattern of the NWs shows four sharp peaks at $35.7^{\circ}, 41.5^{\circ}, 60.1^{\circ}$, and $71.9^{\circ}$, corresponding to (111), (200), (220), and (311) planes of $3 \mathrm{C}-\mathrm{SiC}$, respectively. ${ }^{28}$ Figure 1 (b) shows the SEM image of the original SiC NW bridging on the Au electrodes. After the addition of $\mathrm{NaBH}_{4}$ solution and laser radiation at a fluence of $1.14 \mathrm{~J} / \mathrm{cm}^{2}$ for $5 \mathrm{~s}$, no ablation on $\mathrm{SiC} \mathrm{NW}$ can be observed and the $\mathrm{NW}$-electrode structure is preserved [Fig. 1(c)].

Figures 1(e) and 1(f) show the Raman spectra of 3C-SiC NWs before and after fs laser irradiation. The as-prepared SiC NWs exhibit
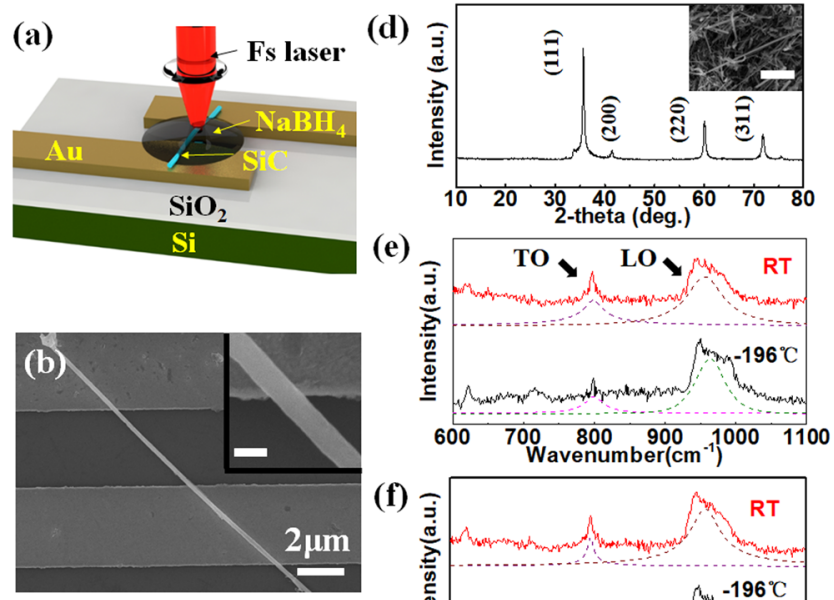

$2 \mu \mathrm{m}$
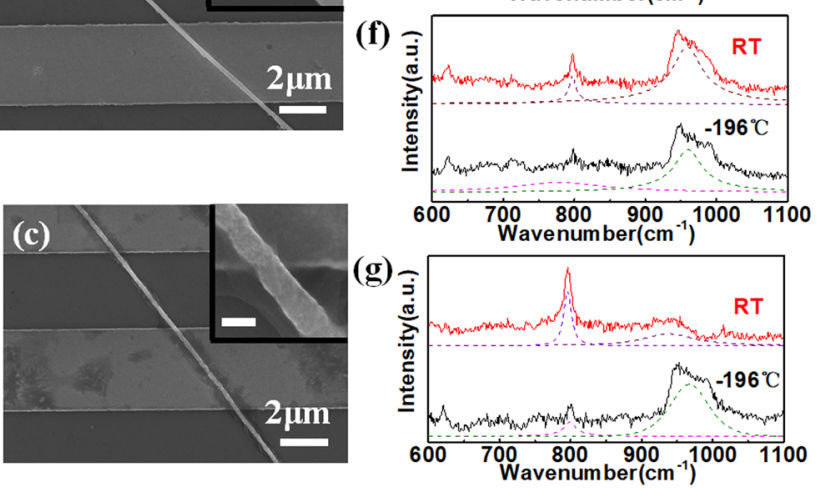

FIG. 1. (a) Schematic of fs laser pointed irradiation on the SiC NW circuit. SEM images of (b) pristine and (c) laser radiated $\mathrm{NaBH}_{4}$ wrapped SiC NWs bridging on Au electrodes. Inset scale bars in (b) and (c): $200 \mathrm{~nm}$. (d) XRD spectrum of the asreceived SiC NWs. The inset in (d) shows the SEM image of SiC NW clusters. Scale bar: $5 \mu \mathrm{m}$. The Raman spectra and their Pearson VII Area fit curves (dashed lines) of (e) pristine SiC NWs, (f) laser irradiated SiC NWs, and (g) laser irradiated $\mathrm{NaBH}_{4}$ wrapped SiC NWs. Temperatures: room temperature (red) and $-196{ }^{\circ} \mathrm{C}$ (black). Laser fluence: $1.14 \mathrm{~J} / \mathrm{cm}^{2}$. Duration: $5 \mathrm{~s}$.

two broad absorption bands. These two peaks at around $800 \mathrm{~cm}^{-1}$ and $960 \mathrm{~cm}^{-1}$ are the dominant features of the $3 \mathrm{C}-\mathrm{SiC}$ crystal, corresponding to the modes of transverse (TO) and longitudinal optical (LO) phonons, respectively. ${ }^{29-31}$ Both peaks display a relatively red-shift $\left(<5 \mathrm{~cm}^{-1}\right)$ when the temperature rose from $-196^{\circ} \mathrm{C}$ to $\sim 25^{\circ} \mathrm{C}$ (room temperature), which can be ascribed to the thermal expansion of the lattice, lattice-mismatch-induced strain, and anharmonic coupling to other photons at increased temperature. ${ }^{32}$ However, the Raman spectrum of laser irradiated $\mathrm{NaBH}_{4}$ wrapped $\mathrm{SiC}$ NWs shows that the increased temperature significantly reduces the absorption of the LO mode and broadens its full-width at half-maximum (FWHM) from $\sim 64$ to $\sim 85 \mathrm{~cm}^{-1}$ [Fig. $1(\mathrm{~g})$ ]. Notably, the carrier density and the spatial distribution of the dopants can change the line shape of the LO phonon plasmon-couple (LOPC) modes. ${ }^{33}$ For semiconductors, the free hole carriers can couple to the longitudinal optical phonons excited by laser waves, which will lead to the LOPC mode and then broaden the linewidth of LO mode. Moreover, the increased active hole carrier concentration and phonon numbers at a higher temperature will enhance the inelastic scattering between substitutional atoms and photons, resulting in a decline of the phonon lifetime. ${ }^{34,35}$ Consequently, increasing the temperature will broaden the linewidth and reduce the peak intensity of the LO mode, besides affecting the 
line positions of TO and LO modes. These peak shifting and broadening are observed in Fig. 1(g), which implies that a considerable number of hole carriers have been generated in the SiC NWs after laser irradiation. Therefore, $\mathrm{B}$ doping in $\mathrm{SiC}$ NWs is expected to occur after laser irradiation on $\mathrm{NaBH}_{4}$ wrapped semiconductors.

To investigate the electrical conduction in $\mathrm{SiC} \mathrm{NW}$ before and after fs laser irradiation, back-gate SiC NWFETs are fabricated. Figure 2(a) shows the typical gate-dependent current vs voltage $\left(\mathrm{I}_{\mathrm{SD}}-\mathrm{V}_{\mathrm{SD}}\right)$ curves of the original SiC NW-Au electrode structure. The original $\mathrm{SiC}$ NWFET shows typical n-type behavior, where $\mathrm{I}_{\mathrm{SD}}$ gradually increases when a positive gate-to-source voltage is applied. The same gate dependence is observed in Figs. 2(b) and 2(c), showing that the type of charged carriers in SiC NWs will not be affected with only $\mathrm{NaBH}_{4}$ added or directly irradiated by the fs laser. As illustrated in Fig. 2(b), the SiC NWs band bends down to bring the Fermi level in line with that of the metal at the contact. When $\mathrm{V}_{\mathrm{G}}>0$, the bands are lowered, there is an accumulation of electrons in n-type $\mathrm{SiC} \mathrm{NWs}$, and the conductivity between $\mathrm{SiC}$ NWs and $\mathrm{Au}$ electrodes is enhanced. ${ }^{36}$ However, the opposite gate dependence $\left(\mathrm{I}_{\mathrm{SD}}-\mathrm{V}_{\mathrm{G}}\right)$ of SiC NWFETs can occur after laser irradiation on $\mathrm{NaBH}_{4}$ wrapped SiC NWs [Fig. 2(d)]. B-element doping in $\mathrm{SiC}$ then tuned the pristine n-type $\mathrm{SiC} \mathrm{NW}$ to p-type, where the band diagram changes differently at the metalsemiconductor contact [inset in Fig. 2(d)]. Since the positive $V_{G}$ will drag the hole carriers apart from the channel between the p-type semiconductor and metal electrodes, conductivity will be reduced, which is also confirmed by the current response chart.

By changing the potential of the back gate in the substrate, the transconductance can be measured, which can be used to determine the doping concentration of the $\mathrm{B}$ dopants. ${ }^{22}$ In the linear transistor regime, the relation between the transconductance $g$ and the carrier mobility $\mu$ is $g=d I_{S D} / d V_{G}=C \mu V_{S D} / L^{2}$. C is the capacitance and $\mathrm{L}$ is the length of the active region. The $\mathrm{SiC} \mathrm{NW-SiO}$-Si capacitance $C=2 \pi L \varepsilon_{0} \varepsilon_{r} / \cosh ^{-1}((R+h) / R)$, where $\varepsilon_{0} \varepsilon_{r}$ is the dielectric
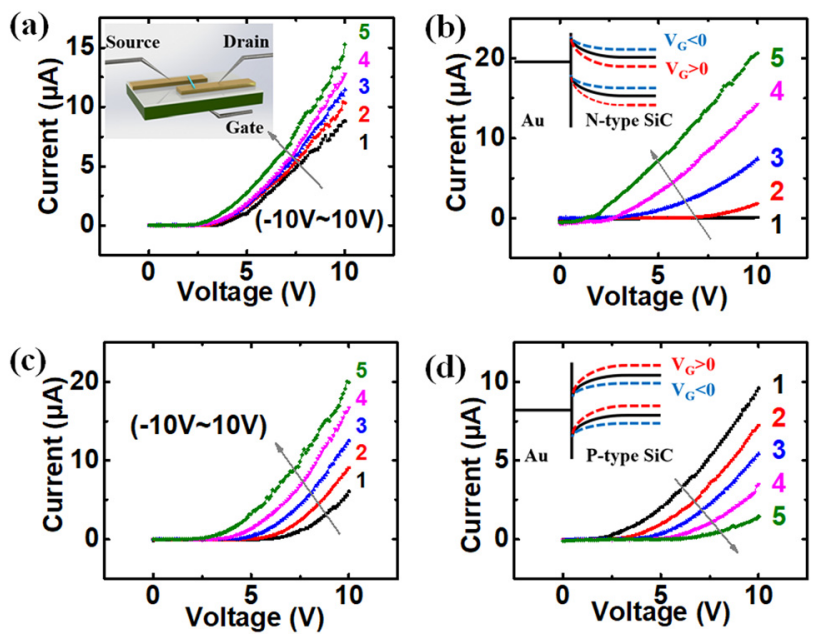

FIG. 2. The current-voltage response of source and drain $\left(I_{S D}-V_{S D}\right)$ under different gate voltages of (a) pristine SiC NWFET and (b) $\mathrm{NaBH}_{4}$ wrapped SiC NWFET without laser irradiation. (c) SiC NWFET after laser irradiation (no $\mathrm{NaBH}_{4}$ added) and (d) $\mathrm{NaBH}_{4}$ wrapped SiC NWFET after laser irradiation. Laser fluence: $1.14 \mathrm{~J} / \mathrm{cm}^{2}$. Duration: $5 \mathrm{~s}$. Insets in (b) and (d) show the schematic of the effect of $V_{G}$ on the electrostatic potential. constant, $\mathrm{R}$ is the radius of $\mathrm{SiC} \mathrm{NW}$, and $\mathrm{h}$ is the thickness of the dielectric layer, corresponding to the $300 \mathrm{~nm} \mathrm{SiO}_{2}$ layer on the $\mathrm{Si}$ substrate. The slopes of $d I_{S D} / d V_{G}$ are calculated as $3.23 \times 10^{-8} \mathrm{~S}$ for pristine $\mathrm{SiC}$ NWs and $4.08 \times 10^{-8} \mathrm{~S}$ for B-doped $\mathrm{SiC}$ NWs. The carrier mobility is then calculated as $9.33 \times 10^{-4} \mathrm{~m}^{2} /(\mathrm{V} s)$ for the pristine $\mathrm{SiC}$ NWs and $1.18 \times 10^{-3} \mathrm{~m}^{2} /(\mathrm{V} s)$ for the B-doped $\mathrm{SiC} \mathrm{NWs}$. For reference, the carrier mobility of highly compensated $4 \mathrm{H}-\mathrm{SiC}$ (mainly with vanadium) was reported as $1 \times 10^{-3} \sim 1 \times 10^{-2} \mathrm{~m}^{2} /(\mathrm{V} s){ }^{37-39}$ At room temperature, the Hall mobility of Boron doped bulk $\mathrm{SiC}$ by ion implantation has a value of $3.6 \times 10^{-3} \mathrm{~m}^{2} /(\mathrm{V} s){ }^{40}$ This means that the fs laser selective doping has a similar doping level of bulk SiC by ion implantation. The conductivity is related to the carrier (doping) concentration $n$ as $\sigma=n q \mu$, where $\sigma$ is the conductivity and $\mathrm{q}$ is the elementary charge. The resistivity was measured as $1.6 \times 10^{-2} \Omega \mathrm{m}$ of B-doped SiC NWs, which means that the conductivity is $62.5 \mathrm{~S} / \mathrm{m}$, and the hole carrier (doping) concentration in B-doped SiC is calculated as $\sim 3.3 \times 10^{17} \mathrm{~cm}^{-3}$.

The ultrafast laser selective site doping can be applied to precisely fabricate segmented n- and p-type on a single NW device by well controlling the laser spot size and positions. Figure 3 illustrates the process of the p-n junction and even p-type fabrication on a single n-type $\mathrm{SiC}$ NW. The I-V curve is symmetrical under forward and reverse biasing, which indicates the homogeneity of the material composition of $\mathrm{SiC}$ NW, as well as the contact state with electrodes [Fig. 3(a)]. After fs laser irradiation is applied to the vicinity of the anode, the B element is expected to be doped into the vacancy sites in SiC NW, resulting in p-type conversion. ${ }^{41,42}$ Therefore, the asymmetrical current response appears in this partially modified NW structure under forward and (a)

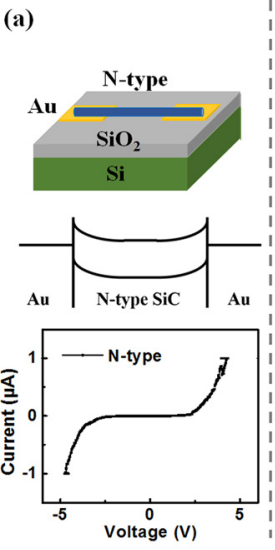

(b)

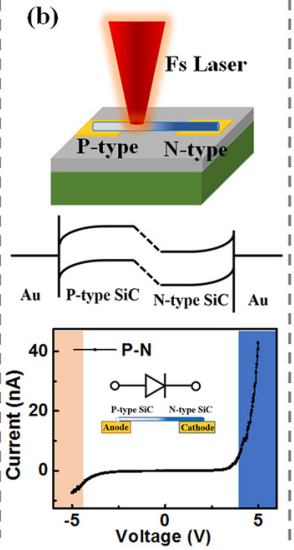

(c)

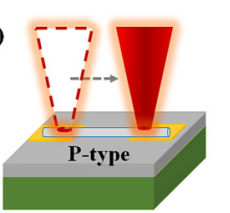

(d)

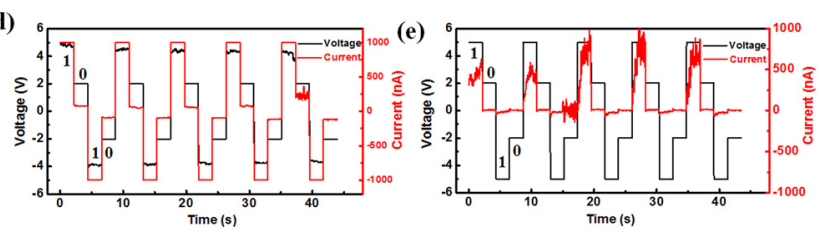

FIG. 3. Electrical responses of pristine and fs laser processed SiC NW homojunction devices. I-V characteristic and related band diagram of (a) original SiC NW on Au electrodes, (b) SiC NW after selective doping at the anode, and (c) SiC NW after doping on the whole structure. The current response of (d) an n-type SiC NW circuit and (e) a single p-n SiC NW circuit under programmed voltage biasing. The voltages applied for different digital signals are $5 \mathrm{~V}, 2 \mathrm{~V},-5 \mathrm{~V}$, and $-2 \mathrm{~V}$. 
reverse biasing [Fig. 3(b)]. The junction between the original n-type part and the doped p-type part can be depleted of charge carriers in the single NW, which allows a flow of electricity in the p-type to ntype direction but not in the opposite direction, resulting in current reduction compared with the pristine n-type structure. Figure 3(c) shows the symmetrical current response after the whole $\mathrm{SiC} \mathrm{NW}$ is processed by fs laser irradiation. With continuous element doping along the fs laser path on $\mathrm{SiC}$, the NW turns from n- to p-n type and finally into p-type. The $\mathrm{Au} / \mathrm{SiC} \mathrm{NW} / \mathrm{Au}$ structure can then be considered as a pair of reverse Schottky diodes, which shows the symmetrical current response with decreased current amplitude under applied bias. ${ }^{43}$ Due to the differential current response of $\mathrm{n}$ - and p-type under forward and reverse biasing, functionalization can be achieved in segmented p-n SiC NW. Figures 3(d) and 3(e) show the current response of n-type and p-n SiC NW under programmed voltages of $5 \mathrm{~V} \rightarrow 2 \mathrm{~V}$ $\rightarrow-5 \mathrm{~V} \rightarrow-2 \mathrm{~V}$. Under forward biasing, the input analog signals with voltages of $2 \mathrm{~V}$ and $5 \mathrm{~V}$ generate the low and high currents, representing digital signals of " 0 " and " 1 ", respectively. As a comparison, the estimated input digital signal representing $0(-2 \mathrm{~V})$ and $1(-5 \mathrm{~V})$ can only receive the output signal with 0 in the single p-n type NW circuit, while the current response behaves the same with input voltages of $2 \mathrm{~V}$ and $5 \mathrm{~V}$. The p-n junction also shows good repeatability under cycled biasing. Notably, site-selective doping of SiC NWs by a focused laser beam will not bring in blunt grain boundaries compared with the direct joining of individual components for multiple $\mathrm{n}$-/p-segment fabrication, which can achieve a combined functional unit with high flexibility. Therefore, more complex elementary units (e.g., n-p-n or p-n-p) can be fabricated using this laser doping method on a single NW.

It is known that under fs laser irradiation, crystalline defects and vacancies can be generated in semiconductor NWs and the dopant molecules are simultaneously decomposed. ${ }^{44}$ The released B acting as a p-type dopant will then diffuse into the vacancy sites of SiC NWs. Under low laser pulse energy, a defect-activation process in $\mathrm{SiC}$ will dominate the laser-matter interaction, while thermal processing is no longer applicable and the incubation effects will take place. ${ }^{45,46}$ After the laser irradiation, the electronic excitation might weaken the interatomic bonding, thus lowering the vibrational energy to produce lattice disorder, ${ }^{47,48}$ which can accelerate the diffusion of doping elements in semiconductors.

Figure 4 shows the fast Fourier transform (FFT) and corresponding inverse Fourier transform (IFT) TEM images of the $\mathrm{SiC}$ NWs before and after fs laser irradiation. The distribution/concentration of point defects in crystals can be obtained by gray analysis of the IFT images. ${ }^{49,50}$ Therefore, the concentration of point defects in pristine $\mathrm{SiC}$ NWs is $<10^{-3}$ from the IFT image in Fig. 4(a), while after laser irradiation at a fluence of $1.14 \mathrm{~J} / \mathrm{cm}^{2}$ for $5 \mathrm{~s}$, the defect concentration increases by tens of times as the gray level of the specific region is significantly reduced from IFT in Fig. 4(b). This tendency confirms the formation of point defects and vacancies in $\mathrm{SiC}$ NWs after fs laser irradiation, thus accelerating the diffusion of $\mathrm{B}$ elements into NWs [Fig. 4(c)]. This defect-activation transversion by nonlinear multiphoton absorption is also applicable to other semiconductor materials, showing the possibility of doping elements in a wide range of materials.

In summary, the fs laser can be used for reliable selective site doping in SiC NW. After fs laser irradiation at a fluence of $1.14 \mathrm{~J} / \mathrm{cm}^{2}$, the
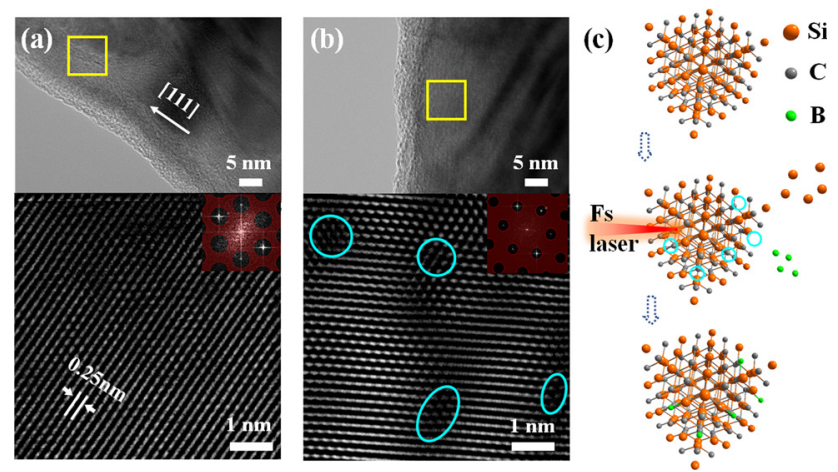

FIG. 4. TEM image and the IFT image in the selected region of SiC nanowires (a) before fs laser irradiation and (b) after fs laser irradiation at a fluence of $1.14 \mathrm{~J} / \mathrm{cm}^{2}$ for $5 \mathrm{~s}$. The concentration of semiconductor point defects is $<10^{-3}$ and $\sim 10^{-2}$ in (a) and (b), respectively. (c) Schematic of fs laser irradiation directed B doping in SiC NWs.

B doped $\mathrm{SiC}$ NWs segment changes from n-type to p-type with a mobility of $1.18 \times 10^{-3} \mathrm{~m}^{2} /(\mathrm{V} s)$ and a hole carrier concentration of $\sim 3.3 \times 10^{17} \mathrm{~cm}^{-3}$. Raman spectroscopy performed on the laser processed SiC NWs shows that the linewidth of the LO-phonon bands increases from $\sim 64$ to $\sim 85 \mathrm{~cm}^{-1}$ and the peak intensity of the LO mode becomes lower at higher temperatures, indicating hole carrier generation in SiC NWs after laser irradiation. Under relatively low laser energy input, crystalline defects and vacancies are formed in $\mathrm{SiC}$ NWs, and the dopant molecules are simultaneously decomposed. The released B acting as a p-type dopant will then be incorporated into the vacancy sites in $\mathrm{SiC}$ NWs. By precisely controlling the laser irradiation energy, functional $n-p$ and p-type (even more complex segments) $\mathrm{SiC}$ NW units are fabricated with a noticeable electrical response. This focused fs laser irradiation directed selective site doping in semiconductor NWs can be utilized to precisely fabricate complex functional NW devices for nanoelectronics.

This work was supported by the National Key Research and Development Program of China (No. 2017YFB1104900) and National Natural Science Foundation of China (Nos. 51520105007 and 51775299). A fruitful discussion with Professor Walt Duley is acknowledged.

\section{REFERENCES}

${ }^{1}$ K. Zekentes and K. Rogdakis, J. Phys. D: Appl. Phys. 44, 133001 (2011).

${ }^{2}$ S. Chen, P. Ying, L. Wang, G. Wei, F. Gao, J. Zheng, M. Shang, Z. Yang, W. Yang, and T. Wu, NPG Asia Mater. 7, e157 (2015).

${ }^{3}$ A. Uzun and K. Teker, Microelectron. Eng. 205, 59 (2019).

${ }^{4}$ T. Yang, X. Chang, J. Chen, K. Chou, and X. Hou, Nanoscale 7, 8955 (2015).

${ }^{5}$ W. Yang, H. Araki, C. Tang, S. Thaveethavorn, A. Kohyama, H. Suzuki, and T. Noda, Adv. Mater. 17, 1519 (2005).

${ }^{6}$ J. Niu, J. Wang, and Q. Xu, Langmuir 24, 6918 (2008).

${ }^{7}$ S. Chen, M. Shang, Z. Yang, J. Zheng, L. Wang, Q. Liu, F. Gao, and W. Yang, J. Mater. Chem. C 4, 7391 (2016).

${ }^{8}$ S. Chen, P. Ying, L. Wang, G. Wei, and W. Yang, "Temperature-dependent field emission of flexible n-type silicon carbide nanoneedle emitters," Appl. Phys. Lett. 13, 133106 (2014).

${ }^{9}$ S. Chen, M. Shang, L. Wang, Z. Yang, F. Gao, J. Zheng, and W. Yang, ACS Appl. Mater. Interfaces 9, 35178 (2017). 
${ }^{10}$ T. Yang, X. Chang, J. Chen, K. Chou, and X. Hou, Nanoscale 7, 8955 (2015).

"S. Chen, L. Zhao, L. Wang, F. Gao, and W. Yang, J. Mater. Chem. C 7, 3173 (2019).

${ }^{12}$ W. Li, Q. Liu, Z. Fang, L. Wang, S. Chen, F. Gao, and X. Fang, Adv. Energy Mater. 9, 1900073 (2019).

${ }^{13}$ T. Yang, L. Zhang, X. Hou, J. Chen, and K. C. Chou, Sci. Rep. 6, 24872 (2016).

${ }^{14}$ L. Fradetal, E. Bano1, G. Attolini, F. Rossi, and V. Stambouli, Nanotechnology 27, 235501 (2016).

${ }^{15}$ Y. Chen, X. Zhang, and Z. Xie, ACS Nano 9, 8054 (2015).

${ }^{16} \mathrm{~F}$. Takano, W. Wang, H. Akinaga, H. Ofuchi, S. Hishiki, and T. Ohshima, J. Appl. Phys. 101, 09N510 (2007).

${ }^{17}$ Z. Tian, N. R. Quick, and A. Kar, Acta Mater. 54, 4273 (2006).

${ }^{18}$ H. Song, S. Kwon, H. Seo, J. Moon, J. Yim, J. Lee, H. Kim, and J. Jeong, Appl. Phys. Lett. 89, 152112 (2006).

${ }^{19}$ A. C. Ford, S. Chuang, J. C. Ho, Y. Chueh, Z. Fan, and A. Javey, Nano Lett. 10, 509 (2010).

${ }^{20}$ S. Dhara, A. Datta, C. T. Wu, Z. H. Lan, K. H. Chen, Y. L. Wang, Y. F. Chen, C. W. Hsu, L. C. Chen, H. M. Lin, and C. C. Chen, Appl. Phys. Lett. 84, 3486 (2004).

${ }^{21}$ B. Pecholt, S. Gupta, and P. Molian, J. Laser Appl. 23, 012008 (2011).

${ }^{22}$ J. Wallentin and M. T. Borgström, J. Mater. Res. 26, 2142 (2011).

${ }^{23}$ A. Cavalleri, C. W. Siders, C. Rose-Petruck, R. Jimenez, C. Tóth, J. A. Squier, C. P. J. Barty, K. R. Wilson, K. Sokolowski-Tinten, M. Horn von Hoegen, and D. von der Linde, Phys. Rev. B 63, 193306 (2001).

${ }^{24}$ N. Misra, L. Xu, Y. Pan, N. Cheung, and C. P. Grigoropoulos, Appl. Phys. Lett. 90, 111111 (2007).

${ }^{25}$ L. Lin, G. Zou, L. Liu, W. W. Duley, and Y. N. Zhou, Appl. Phys. Lett. 108, 203107 (2016).

${ }^{26}$ L. Lin, L. Liu, K. Musselman, G. Zou, W. W. Duley, and Y. N. Zhou, Adv. Funct. Mater. 26, 5979 (2016).

${ }^{27}$ N. Misra, C. P. Grigoropoulos, D. P. Stumbo, and J. N. Miller, Appl, Phys. Lett. 93, 121116 (2008)

${ }^{28}$ J. Chen, Q. Shi, L. Xin, Y. Liu, R. Liu, and X. Zhu, J. Alloys Compd. 509, 6844 (2011).

${ }^{29}$ W. Zhou, X. Liu, and Y. Zhang, Appl. Phys. Lett. 89, 223124 (2006).

${ }^{30}$ M. S. Liu, L. A. Bursill, S. Prawer, K. W. Nugent, Y. Z. Tong, and G. Y. Zhang, Appl. Phys. Lett. 74, 3125 (1999).
${ }^{31}$ R. Gautam, S. Vanga, F. Ariese, and S. Umapathy, EPJ Tech. Instrum. 2, 8 (2015).

${ }^{32}$ M. Bauer, A. M. Gigler, A. J. Huber, R. Hillenbrand, and R. W. Stark, J. Raman Spectrosc. 40, 1867 (2009).

${ }^{33}$ S. Nakashima and H. Harima, J. Appl. Phys. 95, 3541 (2004).

${ }^{34}$ P. Mishra and K. P. Jain, Phys. Rev. B 62, 14790 (2000).

${ }^{35}$ Y. Chen, X. Zhang, Q. Zhao, L. He, C. Huang, and Z. Xie, Chem. Commun. 47, 6398 (2011).

${ }^{36}$ Y. Cui, X. Duan, J. Hu, and C. M. Lieber, J. Phys. Chem. B 104, 5213 (2000).

${ }^{37}$ W. C. Mitchel, R. Perrin, J. Goldstein, A. Saxler, M. Roth, S. R. Smith, J. S. Solomon, and A. O. Evwaraye, J. Appl. Phys. 86, 5040 (1999).

${ }^{38}$ S. Kagamihara, H. Matsuura, T. Hatakeyama, T. Watanabe, M. Kushibe, T. Shinohe, and K. Arai, J. Appl. Phys. 96, 5601 (2004).

${ }^{39}$ F. La Via, G. Galvagno, F. Roccaforte, A. Ruggiero, and L. Calcagno, Appl. Phys. Lett. 87, 142105 (2005).

${ }^{40}$ T. Troffer, M. Schadt, T. Frank, H. Itoh, G. Pensl, J. Heindl, and M. Maier, Phys. Status Solidi (a) 162, 277-298 (1997).

${ }^{41}$ X. Zhang, J. R. Ho, and C. P. Grigoropoulos, Int. J. Heat Mass Transfer 39, 3835 (1996).

${ }^{42}$ D. Kim, S. Hong, K. Jeong, C. Lee, H. Kim, and M. Cho, ACS Nano 13, 1683 (2019).

${ }^{43}$ S. Xing, L. Lin, G. Zou, L. Liu, P. Peng, A. Wu, W. W. Duley, and Y. N. Zhou, Nanotechnology 28, 405302 (2017).

${ }^{44}$ E. Kim, C. Ko, K. Kim, Y. Chen, J. Suh, S. Ryu, K. Wu, X. Meng, A. Suslu, S. Tongay, J. Wu, and C. P. Grigoropoulos, Adv. Mater. 28, 341 (2016).

${ }^{45}$ Y. Dong, C. Zorman, and P. Molian, J. Micromech. Microeng. 13, 680 (2003).

${ }^{46}$ Y. Dong and P. Molian, Appl. Phys. A 77, 839 (2003).

${ }^{47}$ H. W. K. Tom, G. D. Aumiller, and C. H. Brito-Cruz, Phys. Rev. Lett. 60, 1438 (1988).

${ }^{48}$ S. V. Govorkov, I. L. Shumay, W. Rudolph, and T. Schroder, Opt. Lett. 16, 1013 (1991).

${ }^{49} \mathrm{X}$. Zhang, X. Zhao, and J. Chong, J. Chongqing Univ. 31, 139 (2008).

${ }^{50}$ H. Qiu, T. Xu, Z. Wang, W. Ren, H. Nan, Z. Ni, Q. Chen, S. Yuan, F. Miao, F. Song, G. Long, Y. Shi, L. Sun, J. Wang, and X. Wang, Nat. Commun. 4, 2642 (2013). 